\title{
A Novel Method for Bacterial UTI Diagnosis Using Raman Spectroscopy
}

\author{
Evdokia Kastanos, ${ }^{1}$ Alexandros Kyriakides, ${ }^{2}$ Katerina Hadjigeorgiou, ${ }^{2}$ and Costas Pitris ${ }^{2}$ \\ ${ }^{1}$ Department of Life and Health Sciences, University of Nicosia, 1700 Nicosia, Cyprus \\ ${ }^{2}$ Department of Electrical and Computer Engineering, University of Cyprus, 1678 Nicosia, Cyprus
}

Correspondence should be addressed to Costas Pitris, cpitris@ucy.ac.cy

Received 16 July 2011; Accepted 19 September 2011

Academic Editor: Jin Zhang

Copyright (C) 2012 Evdokia Kastanos et al. This is an open access article distributed under the Creative Commons Attribution License, which permits unrestricted use, distribution, and reproduction in any medium, provided the original work is properly cited.

\begin{abstract}
The current state of the art on bacterial classification using Raman and Surface Enhanced Raman Spectroscopy (SERS) for the purpose of developing a rapid and more accurate method for urinary tract infection (UTI) diagnosis is presented. SERS, an enhanced version of Raman offering much increased sensitivity, provides complex biochemical information which, in conjunction with advanced analysis and classification techniques, can become a valuable diagnostic tool. The variety of metal substrates used for SERS, including silver and gold colloids, as well as nanostructured metal surfaces, is reviewed. The challenges in preprocessing noisy and complicated spectra and the various methods used for feature creation as well as a novel method using spectral band ratios are described. The various unsupervised and supervised classification methods commonly used for SERS spectra of bacteria are evaluated. Current research on transforming SERS into a valuable clinical tool for the diagnosis of UTIs is presented. Specifically, the classification of bacterial spectra (a) as positive or negative for an infection, (b) as belonging to a particular species of bacteria, and (c) as sensitive or resistant to an antibiotic are described. This work can lead to the development of novel technology with extremely important benefits for public health.
\end{abstract}

\section{Introduction}

Urinary tract infections (UTIs) are some of the most common types of infections in humans with an estimated 34 percent of adults aged 20 or older reported as having had at least one occurrence of a UTI or cystitis. Specifically, over $50 \%$ of women and over $13 \%$ of men will have a UTI at least once in their lifetime $[1,2]$. This results in millions of doctors' visits and a cost of several billion USD every year just in the USA alone [3, 4]. UTI diagnosis is a multistep process which includes the determination of the concentration of pathogens, and the identification of the responsible bacteria, as well as their susceptibility to various antibiotics, the socalled antibiogram. Such assays require repeated culturing of a sample and take over 48 hours in order for bacterial colonies to be grown, counted, and exposed to antibiotics using conventional clinical methods. Since the patient cannot remain untreated during this rather prolonged period before definitive diagnosis is obtained, physicians prescribe broad spectrum antibiotics prior to antibiogram results. This practice has many undesirable consequences, both short term and long term: (i) unsuccessful treatments leading to chronic infections, (ii) increased health care costs, and (iii) increased antibiotic resistance by a growing number of bacterial strains [5-8]. Given these concerns, it is obvious that a rapid and accurate method of UTI diagnosis and bacterial susceptibility to antibiotics would offer significant health benefits. Such methodologies are currently being developed and include the promising application of Raman spectroscopy for the diagnosis of UTIs.

Recently, rapid diagnosis methods based on PCR have been developed in order to bypass the need for culturing [9] as well to identify genes that confer antibiotic resistance [10]. Although such PCR assays are fast and very sensitive, they typically require species and strain-specific probes that may or may not be available for a particular organism. Additionally, amplification methods like PCR suffer from contamination problems, complex interpretation of results, as well 
as high costs. Mass spectrometry is another method that has been proposed as an alternative approach for bacterial diagnostics without culturing [11]. However, like the PCR approach, mass spectrometry also depends on prior knowledge of the pathogen under study and suffers from increased complexity and cost.

Vibrational spectroscopies, like Raman spectroscopy, have been used, for the last few years, to detect bacteria with minimal sample manipulation [12-14]. Classification of bacterial species, as well as subspecies, has been achieved with great accuracy and speed, especially with surface-enhanced Raman spectroscopy (SERS) [15] which allows enhancement of the inherently weak Raman signal. More recently, it was shown that bacterial susceptibility to antibiotics can also be determined using Raman spectroscopy and SERS [16-18].

1.1. Raman Spectroscopy. Whole organism fingerprinting is a relatively recent approach for the identification of pathogens based on their unique chemical characteristics with minimal sample preparation [19]. Some of the techniques that are being evaluated for such fingerprinting include vibrational spectroscopic methods such as Fourier-Transform Infrared spectroscopy (FTIR), Raman Spectroscopy, and UV resonance Raman spectroscopy (UVRR). Vibrational spectroscopic features arise from the loss or gain of energy by photons which are inelastically scattered from a vibrating sample. This energy change results in an alteration of the photons' wavelength which in the case of organic molecules is directly related to the vibrational states of its chemical bonds. Therefore, quantitative biochemical information can be extracted from the spectra.

The Raman effect is observed when a very small number of photons incident on a molecule (about 1 in $10^{7}$ ) are inelastically scattered, that is, scattered at different frequencies than the frequency of the incident photons [20]. A Raman spectrum is a plot of the intensity of scattered light versus the energy difference between the incident photons and the Raman-scattered photons, and contains information about the chemical composition and the molecular structure of a substance. A major limitation of Raman is the weakness of the Raman effect which results in very low signals, often below the limit of detection for dilute biological samples. In addition, Raman spectroscopy is not very selective with respect to the various molecules present in complex biological samples, resulting in highly congested spectra. Finally, Raman spectroscopy suffers from a strong fluorescence background which significantly decreases the dynamic range of the measurement. FTIR, on the other hand, is much more sensitive than Raman, but its use in biological systems is very limited due to the strong absorbance of water at those wavelengths. However, several publications have come out in the last few years that show discrimination of bacteria using FTIR and also quite complex classification analysis methods [21, 22]. UVRR is also more sensitive than normal Raman and does not suffer from high fluorescence background. It has already been exploited for the discrimination of UTI bacteria [23]. The highly energetic nature of UV photons, however, can cause photochemical or burning effects on a sample and limits its use as a routine method for bacterial analysis. Additionally, excitation in the UV is not very useful for the discrimination of different species of bacteria as it mostly identifies nucleic acid bases, which are not expected to vary in abundance between different bacterial species. The widespread adoption of UVRR is also limited by the high cost and complexity of the equipment required.

Raman spectroscopy has also been successfully applied to bacterial identification in the configuration of a Raman microscope, which allows collection of spectra from single cells and enhances sensitivity. The group of Hutsebaut et al. have used Raman microscopy to identify samples belonging to 3 different Bacillus species [24]. Xie et al. successfully classified 6 different bacterial species [25]. Studies by Harz et al. [26] have shown that discrimination of different Staphylococcus species can be achieved with 95-97\% accuracy not only at species but also at strain level. The same group has successfully discriminated 29 strains of bacteria derived from a variety of species [27]. Despite increased sensitivity, studies using micro-Raman spectroscopy have to face a few unique challenges. First, the fact that imaging individual bacteria is a time consuming process that leads to inadequate evaluation of large samples. Second, the increased variability between spectra of individual cells, mostly due to variations in the growth stage of each cell, usually dictates a need for more complex classification methods.

1.2. Surface-Enhanced Raman Spectroscopy. Surface Enhanced Raman Spectroscopy (SERS) is a variation of Raman spectroscopy which offers significant enhancement of the signal (up to $10^{14}$ times), thus making detection faster, simpler, and more accurate. The enhancement is a result of the effect of plasmon resonance, that is, the unison oscillation of electrons on the surface of a metallic nanostructure as a result of incident light of the right, resonant, frequency. These oscillations produce an enhanced electromagnetic field in the proximity of the surface. If a sample is within a few nanometers $(\mathrm{nm})$ from the nanostructure, it will experience this enhanced field and exhibit a stronger Raman signal [2831]. The enhancement is such that even single molecules can be detected. However, this enhancement rapidly decreases as the distance between the target sample and the nanoparticle increases and completely disappears within $10-20 \mathrm{~nm}$ of separation. For a more detailed discussion of SERS mechanisms, the reader can refer to an extensive volume of literature [28-31]. SERS spectra also exhibit reduced data congestion compared to normal Raman spectra, since the enhancement is significant only for molecules found on, or very close to, the SERS substrate. SERS also significantly improves the dynamic range of the measurement due to the ability of SERS-active substrates to quench fluorescence. Finally, SERS can be performed using simple, mobile, and relatively inexpensive equipment providing a significantly improved signal at high speed and low cost. SERS offers significant advantages for the identification of bacteria species and bacterial function. (a) The enhancement of the Raman signal allows accurate detection of minute quantities of bacteria, therefore, eliminating the need for time and resource 
consuming culturing processes. (b) In the case of microRaman spectroscopy, the enhancement offered by SERS allows identification of even individual bacteria which enhances the selectivity of the identification in the case of samples with multiple constituents. (c) Metal nanoparticles, under certain conditions, are internalized by the bacteria thus providing signal enhancement from both external (cell wall) molecules as well as the internal milieu of the bacterium [32]. This is an important advantage since the sensitivity and selectivity of bacterial classification is significantly improved.

1.2.1. SERS Substrates for Classification of Bacteria. One of the first groups to work on the identification of bacteria by SERS is the group of Efrima et al. [33-36]. The SERS-active substrate they employed was not only silver but also gold colloid. What is unique about their methodology is that they usually produced the colloid in the presence of the bacteria and not mixing pre-formed colloid with the sample. In addition, they demonstrated growth of colloids in the interior or the exterior of bacterial cells obtaining different spectra in each case. As far as the collection of SERS spectra is concerned, they have done it under a large variety of conditions, including various preparations of their substrates, using different fractions of bacterial cells, and different excitations, ranging from the UV to the NIR $(250-800 \mathrm{~nm})$. Jarvis et al. is another group who have done a lot of work on SERS and identification of bacteria, and specifically UTI involved bacteria, using silver colloids in combination with different types of Raman microscopes [32, 37-40]. Their group also reported SERS from both intracellular and extracellular bacterial locations [32]. In addition, they were one of the first groups to successfully apply various advanced classification techniques to the SERS spectra. The group of Premasiri et al. have used gold-cluster-covered silicon substrates and a Raman microscope to discriminate SERS spectra of bacteria [41] as well as to classify the bacteria down to the subspecies level [42]. Other groups have shown very high specificity and sensitivity in classification of bacteria, even at the strain level, using silver nanorod arrays as a substrate [43]. Metal nanosurfaces containing various structures have also been used, and some are commercially available like Klarite (Mesophotonics). Klarite gold nanosurfaces do not offer great enhancement for bacteria, however, as the pits of the structure, where most of the enhancement would be expected, are only about $100 \mathrm{~nm}$ in diameter and do not fit most bacteria. A very recent study by Walter et al. uses silver colloids and a microfluidic device and greatly minimizes sample volume and exposure time for obtaining SERS spectra of E. coli, which are classified with high accuracy [44]. A major limitation of SERS in the identification of bacteria are the spectral variations present, even between spectra from the same sample, since the technique is very sensitive to substrate, sample preparation, as well as local field conditions. Despite these limitations SERS, is definitely much more sensitive for identifying and classifying bacteria than normal Raman and is now the preferred route for developing a rapid method of diagnosis of a UTI.

\section{Data Processing, Classification, and Evaluation}

Raman spectra from simple and concentrated solutions, of single or few chemical constituents are easy to measure and interpret. However, as the concentration decreases or the samples become more complicated, for example, tissue or bacteria, the extraction of useful information from the weak and noisy Raman spectra becomes more challenging. The two major factors, which make Raman signals difficult to classify, are the fact that they are usually obscured by a large amount of noise and that the signals have a great deal of variance even between spectra which originate from the same sample. Both of these problems (noise and high variance) must be rigorously addressed when attempting to classify the Raman spectra of UTI involved pathogens.

Classification is the exercise of assigning a class to an object. In the case of analyzing Raman spectra for UTI, the objects are the Raman spectra themselves, and the class of each spectrum is the species or subspecies of the bacteria that yielded the specific measurement. The procedure for performing classification can be broken down into four main steps. The first step is preprocessing which attempts to remove noise and to normalize the Raman spectra. The second step is feature creation which uses the denoised and normalized spectra in order to create features which will be fed to the classification algorithm. In statistical terms, the features can be considered to be the "independent variables." The "dependent variable" is the class. The third step in the classification process is the use of the features by the classification algorithm to yield the estimated class of the sample. The final step is the evaluation of the classification to determine its accuracy.

Although these four steps describe the overall picture of the classification process well, it is not always the case that all steps are necessary or even separable. For example, in some procedures, the denoising, normalization, and feature creation can be conceptualized as one step. Furthermore, in one of the most successful techniques presented for the classification of Raman spectra [45], no explicit normalization is performed in the preprocessing step, but instead, the normalization is accomplished by the distance metric used to compare the feature vectors.

\subsection{Preprocessing}

2.1.1. Noise Removal. The weak nature of the Raman effect results in spectra with very low signal-to-noise ratio (SNR). Noise in Raman spectra takes one of three forms: (i) highfrequency noise, (ii) low-frequency noise, and (iii) cosmic spikes (Figure 1).

High frequency noise comes from the acquisition electronics and other sources of system variation. Median filtering can be used to remove this type of noise [26]. Another technique commonly used is wavelet-based denoising [46]. Both of these techniques work well, and using at least one of them is recommended. Low-frequency noise arises from ambient light entering the spectrograph and fluorescence emission from the sample which, in the case of biological 


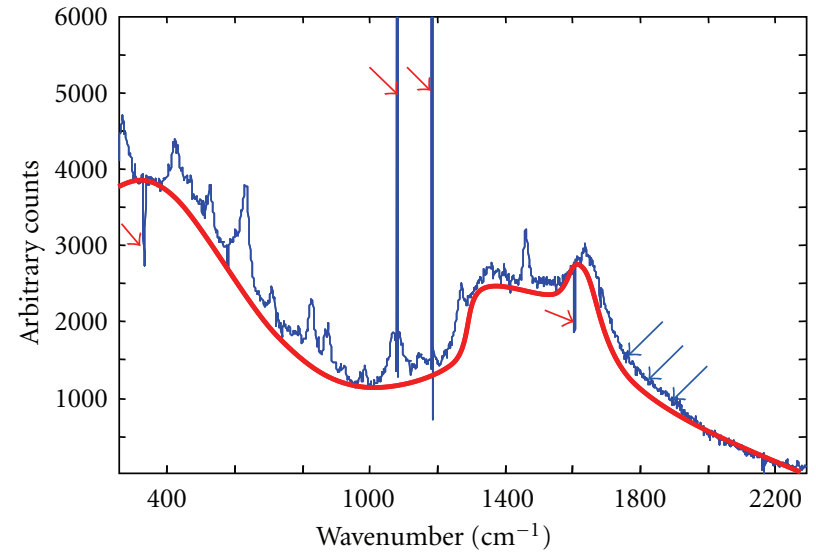

(a)

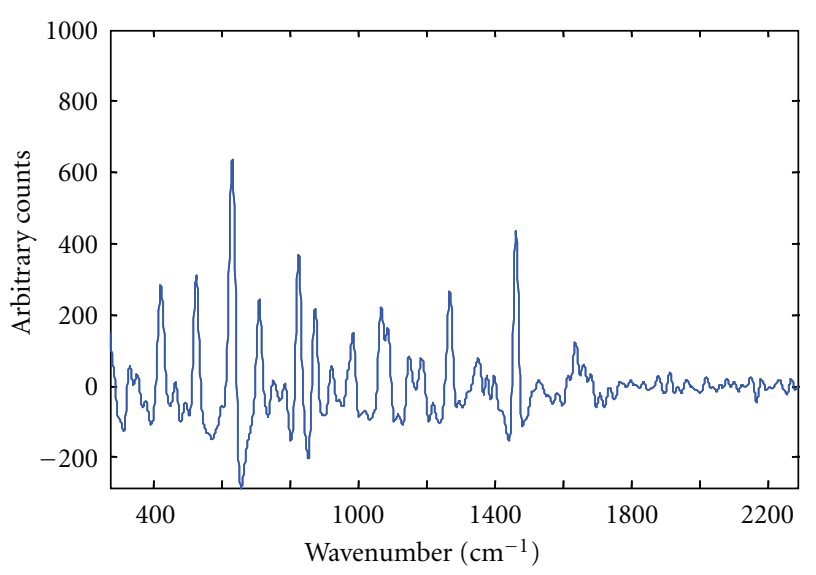

(b)

Figure 1: (a) Raman spectrum of fructose exhibiting cosmic spikes (red arrows), background fluorescence (red curve), and high-frequency noise (blue arrows). (b) The same spectrum after preprocessing.

samples, can significantly reduce the dynamic range of the measurement. This is seen as a background baseline which is present in the spectra. Iterative curve fitting or low pass filtering can be used to estimate and remove the background baseline [20]. Removing the low-frequency noise can help improve the accuracy of the classification, but it is not always necessary. Using the first and second derivatives of the spectrum as features for the classification by itself removes the baseline since the derivatives indicate changes and curvature and not background (see Section 2.2 "Feature Creation" below). Cosmic spikes are spurious, very narrow, spikes appearing in the Raman spectra. They are an artefact of the detection electronics. The techniques used to filter out highfrequency noise can also be used to remove cosmic spikes. For example, it has been shown that median filtering is an effective way to remove this type of noise too [17]. Cosmic spikes can produce outliers which can be detrimental to the accuracy of several classification methods. It is therefore highly recommended that cosmic spikes are always removed before initiating any classification process.

2.1.2. Normalization. Raman spectra acquired sequentially or intermittently from exactly the same sample can exhibit a large amount of variance. This is usually in the form of an additive or multiplicative shift. These variations, in certain cases, can adversely affect the classification and should be eliminated by normalizing the spectra. Two of the most common techniques used are normalization using the highest peak [16] and vector normalization [47]. In the first case, all the spectra are modified so that they have the same minimum and maximum values. In the case of vector normalization the samples are considered to be multidimensional vectors and are modified so that their magnitudes are equal.

2.1.3. Considerations and Caveats. It is important to note that the preprocessing step greatly affects the accuracy of the classification results. A sufficient amount of preprocessing must be performed in order to increase the classification accuracy. At the same time, however, it is imperative to understand that too much preprocessing can introduce user bias and overfitting effects, which will decrease the accuracy of the results when truly unknown samples are subsequently classified. A classification algorithm, which is self-normalizing [45] avoids the pitfalls of too much preprocessing and therefore improves the classification accuracy of the technique.

2.2. Feature Creation. Once the noise removal and normalization have been completed, features must be created to be subsequently used by the classification algorithm. The most common technique of feature creation is Principal Component Analysis [48], which uses a linear transformation to represent the original data into a new coordinate space. The dimensions of this new space are in the directions of maximum variance of the features. The first principal component captures the dimension of maximum variance; the second principal component captures the dimension of the second greatest variance, and, similarly, the subsequent principal components capture the dimensions of successively decreasing variances. It is common to choose only a subset of these principal components as features. Usually, the first few principal components are used, although this is not always the best approach [49]. An important benefit of selecting only a subset of the principal components is that the dimensionality of the data is reduced. This can be advantageous for the classification process for several reasons. The reduced number of features might capture the differences between classes more effectively, thus increasing the classification accuracy. In addition, the reduced number of variables might decrease the complexity of the classification, lower the computational cost, and improve the speed of the classification procedure. In methods such as Discriminant Analysis (DA, discussed below), dimensionality reduction is sometimes required in order to remove features which are highly correlated. In the DA algorithm, such features produce a singular matrix with no inverse, and thus DA cannot be performed. 
Using the first and second derivatives is also a commonly used technique [50-52] for feature creation. This method has the advantage of capturing the successive intensity differences of the Raman spectrum (first derivative), or the successive rates of change of the differences in the spectrum (second derivative). These measures have proven to be very successful in the analysis of Raman data and have been widely adopted. An additional advantage of taking the derivatives of the spectrum is that the baseline is removed without introducing bias or human error which would occur if the baseline was removed manually.

Another technique, which was shown to be successful in feature creation, is the use of spectral band ratios [45]. Each spectrum is broken down into segments, each consisting of a small band of wavenumbers. The mean intensity of each segment is found, and the ratio of the mean intensity of each segment to every other segment in the spectrum is then calculated. These ratios are then used as features for the classification of the data. This technique has two significant advantages. First, normalization is inherently achieved since the intensity of one part of the spectrum is compared to the intensity of other parts of the same spectrum. In this way, even if the intensity of the whole spectrum uniformly varies, the ratios of intensities remain the same. Second, the number of features is much less than the original number of data points thus achieving a reduction in the dimensionality of the data. The success of this method implies that classification can still achieve good accuracy even when using a low-resolution spectrum, an important deduction supporting the use of clinically viable, commercial, low cost, and low-resolution systems.

2.3. Classification. Accurate classification of Raman spectra is crucial if the technique is ever to have a clinical application and, more importantly, impact disease diagnosis and prognosis. The range of classification techniques in the literature is extensive. However, certain methods appear to perform well when applied to Raman spectra, and those will be covered in this section. Classification can be either supervised or unsupervised. Unsupervised classification methods group the data points into clusters without using any information about the class (label) of each data point. Supervised classification methods on the other hand require a set of data points which are labeled, that is, their class is known a priory. These labeled data points are used to train the algorithm, using the known information about the class (label) of each point, to create a model of the data. Once the supervised model has been trained, it can be used to classify data points which were not in the training set, by assigning them to one of the classes described in the training set.

2.3.1. Unsupervised Classification (Clustering). In Raman spectroscopy, hierarchical cluster analysis is the most commonly used unsupervised classification method [53]. Being an unsupervised method, it does not require a priori labeling of the data points. During the training phase, clusters are formed and, subsequently, HCA can be used to classify a new data point by determining into which cluster this new data point falls. The results of HCA are easy to interpret and can be easily visualized as a dendrogram (Figure 3). This ease of interpretation has made HCA very popular. However, this method does not provide good classification performance on data points, which are "out-of-sample," that is, not in the training set. HCA is relatively unstable because clusters formed in the lower levels of the hierarchy can constrain the clusters formed at higher levels of the hierarchy. This constrain can make the analysis unreliable.

The most important consideration when performing cluster analysis is the similarity metric (distance metric), which is used to compare data points. The most commonly used distance metric is the Euclidean distance, but this might not be the most appropriate in the case of Raman spectra. A general disadvantage of cluster analysis is the assumption that clusters do actually exist. This assumption is not always true. Furthermore, it is not always clear how many clusters should be formed. The number of clusters is a prominent concern when performing this type of analysis.

2.3.2. Supervised Classification. In supervised classification, the goal is to assign data points, which were not seen before, into classes learned from a training set assuming that each data point belongs to one of the known classes. For example, Raman spectra obtained from bacteria can be labeled and classified based on the species of the bacterium from which they originated. Each spectrum is a data point, and it belongs to one class. Supervised learning requires that the class of some data points is known (labeled data points). Those points are used as a training set to create a model which can then be used to predict the label of unlabeled data points ("out-of-sample" data points) whose class is not known. Semisupervised methods, which do not require all data points to be labeled, do exist. However, in this paper, we will only deal with supervised methods in which all the data points are labeled. Some of the most commonly used supervised learning methods, applicable to the classification of Raman spectra, are listed below including a brief discussion of their advantages and disadvantages.

Nearest Neighbor. The nearest neighbor approach is very simple and does not explicitly require any training. The data points in the training set are used to predict future unlabeled data points by finding the distance between unlabeled data points and the labeled data points in the training set. The unlabeled data point is predicted to belong to the class of the data point in the training set to which it is closest. In the simplest case, the class is determined by the one data point which is closest, the so-called 1-nearest neighbor (1NN). It is possible to perform the classification using more nearest neighbors. For example, if the three data points in the training set closest to the unlabeled data point are used, then the approach is called 3-nearest neighbor ( $3 \mathrm{NN})$. In the general case, when $\mathrm{K}$ nearest neighbors are used, the approach is called K-nearest neighbor (KNN). A disadvantage of this method is that the prediction could be slow because unlabeled data points must be compared to all data points in the training set. Therefore, the prediction speed depends on 


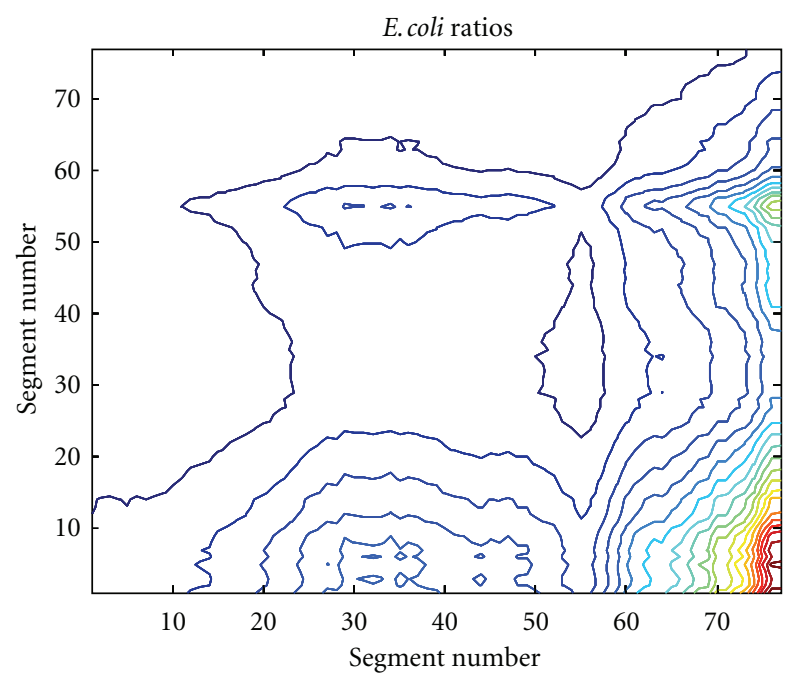

(a)

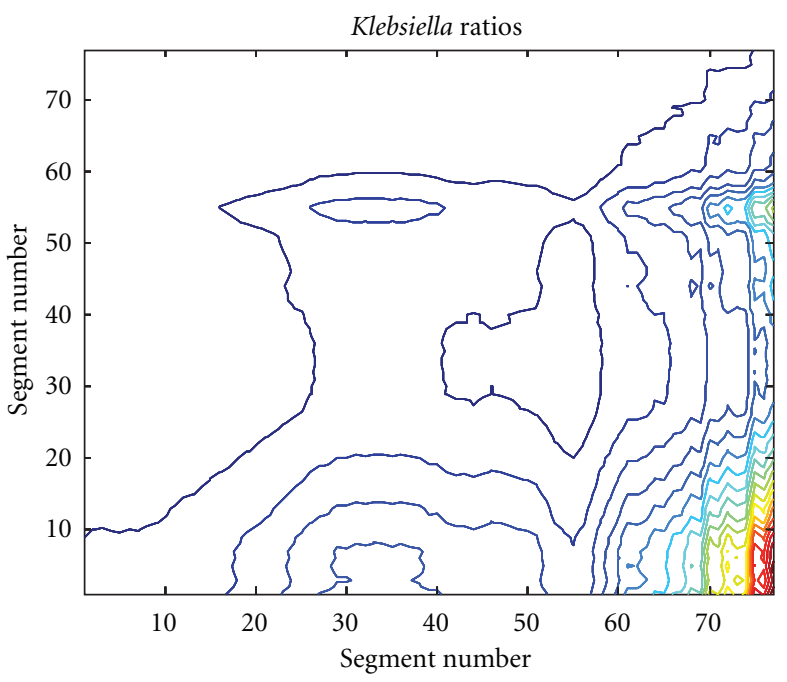

(b)

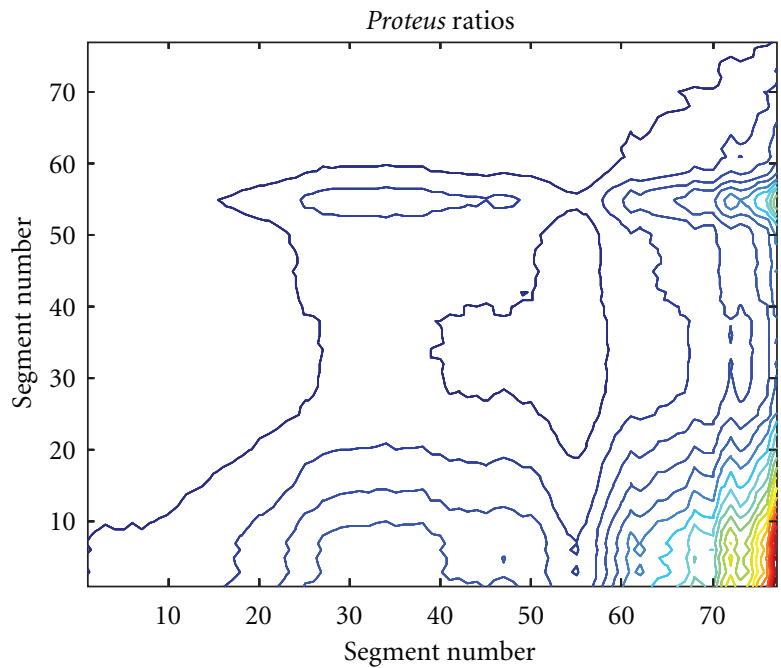

(c)

FIGURE 2: Contour plots of the ratios between different segments of the average Raman spectra from several samples of E. coli, Klebsiella, and Proteus. Differences between the species are evident.

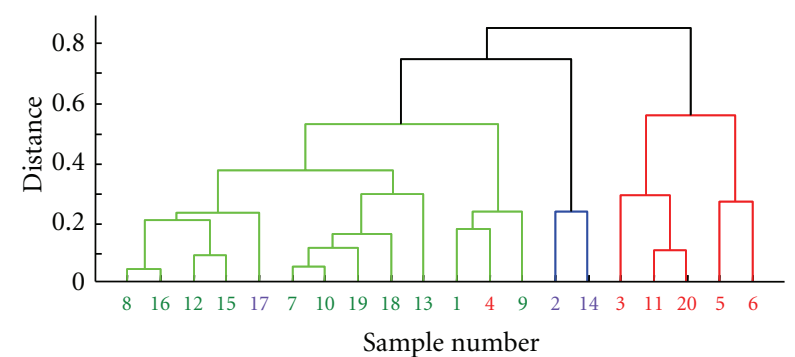

Figure 3: Example of a dendrogram clustering 20 samples according to their "color" property. Below is the sample number indicating the true "color" of each sample. Notice that samples 17 and 4 have been misclassified.

the size of the training set. KNN models have been shown to work well in certain applications although the $3 \mathrm{NN}$ approach is usually preferred. It is a very simple technique to implement. The most serious disadvantage of this method is that it is very sensitive to outliers. It is also very sensitive to irrelevant features which might be present in the data points.

Discriminant Analysis. Linear discriminant analysis (LDA) is one of the most commonly-used classification methods, mainly due to its simplicity and low computational cost [54]. It is a parametric technique which assumes that the independent variables (features) follow a multivariate normal distribution. The training data is used to find the parameters of the normal distribution which describes each class. These distributions are then used to create a discriminant model. In LDA, the assumption is that the different classes are described by identical covariance matrices. If this assumption is removed, then the different classes are allowed to have different covariances. In this way, the separating functions become 
quadratic, and this is called Quadratic Discriminant Analysis (QDA). It is important to choose either LDA or QDA depending on the characteristics of the data. DA is highly sensitive to outliers since they can greatly affect the shape of the calculated distributions. If it is obvious that a data point is an outlier it is best to remove it before carrying out DA. DA is a simple technique that only performs well on data points, which are well separated, and on features which follow a normal distribution. If one of the independent variables (features) is highly correlated with another, or is a function of another set of features, then the calculations for finding the discriminant function will fail. This case is very common when the number of features (independent variables) is much greater than the number of observations (data points). For this reason, it is better to first transform the features before applying DA. The two most commonly used methods for transforming the Raman data are Principal Component Analysis (PCA) and Partial Least squares (PLSs). This leads to the two classification methods known as PC-DFA (Principal Component Discriminant Function Analysis) and PLS-DA (Partial Least Squares Discriminant Analysis).

Principle Components Discriminant Function Analysis (PC-DFA) uses Principal Component Analysis to first transform the data into a new space in order to maximize the variance in each dimension before performing DA. Each dimension is called a principal component. This method can often lead to good results. However, in cases where the variance of the features is not a good criterion for class separability, it is best to use another method, such as PLS-DA.

Partial Least Squares Discriminant Analysis (PLS-DA) is a method which attempts to increase the separation of groups (classes) by representing the data points in a new space [55]. The key difference between PLS and PCA is that PCA aims to find directions of maximum variance, whereas PLS aims to find directions which maximize group separability. In some cases, the two coincide, that is, when group separability is well described by the variance of the features. In other cases, it is best to use PLS instead of PCA. An advantage of PLS-DA is that it can describe complex relationships between features [56]. However, it has been shown that PLS-DA has problems with overfitting [57]. For this reason, it might produce good results when performing cross validation, but it is accuracy on "out-of-sample" data can be low when overfitting on the training data occurs. This is a good example of how crossvalidation accuracy fails to capture the expected classification accuracy on future "out-of-sample" data.

Support Vector Machines (SVMs). A Support Vector Machine is a binary classifier which aims to distinguish between two classes of instances by finding the maximum separating hyperplane between them [58]. For this reason it tends to generalize better than the much simpler DA-based approaches. By design, SVMs can only discriminate between two classes. In order to allow for the classification of more than two classes, one can employ more than one SVMs. SVMs, in their simple form, are linear classifiers. It is possible however to create nonlinear SVMs by increasing the dimensionality of the feature space by using the so-called "kernel trick" $[59,60]$, which employs a kernel function to transform the data. It is thus possible to find a separating hyperplane in higher dimensions where such a hyperplane would not exist in lower dimensions. There are many choices for the type of kernel function to use [60]. The standard choices are the linear kernel (dot-product kernel), the polynomial kernel and the Gaussian Kernel. The best results can be obtained by using an appropriate kernel. It has been shown that for the classification of Raman Spectra, the correlation kernel [45] can give high accuracy on "out-of-sample data."

2.4. Evaluation of the Classification Results. In order to evaluate the performance of a classifier, the most commonly used techniques are cross-validation and performance on a "holdout set." Cross-validation uses only the training set which is broken down into smaller parts, or "folds." It is often preferred to use 10 folds to perform so-called 10 -fold crossvalidation. All the folds, except one, are used for training. Testing consists of predicting the class of each data point in the fold which was left out. The cross-validation procedure performs training and testing as many times as there are folds. Each time, a different fold is left out for testing. In this way, a prediction is made on the class of all the data points in the training set. In the extreme case, each fold can consist of only one data point. This is called Leave-One-Out Cross Validation and is most often used when the training set is small. Cross-validation generally results in a good estimate, but this is not always the case. Classification algorithms which do not generalize well can sometimes perform well in crossvalidation and not so well on "out-of-sample data" [45]. For this reason it is best to test the classification on a "hold-out set" consisting of data points which were not used for training but whose class label is known. The performance of the classifier on such a test set is a good indication of the performance on future unseen ("out-of-sample") data.

\section{Raman Spectroscopy for UTI Diagnosis}

For Raman spectroscopy to become a viable clinical tool, the following key diagnostic objectives must be addressed and successfully accomplish:

(1) classification of urine samples as positive or negative for UTI based on the bacterial load;

(2) identification of the pathogen involved in the positive samples;

(3) determination of the antibiogram, that is, antibiotic sensitivity, of the bacteria involved.

Recent research results indicate that it may indeed be possible to achieve all three of the above objectives with Raman or SERS offering rapid and accurate UTI diagnosis using lowcost, turn-key equipment.

3.1. Classification of Bacterial Samples as Positive or Negative. Most of the work published on Raman spectroscopy of bacteria has focused on identification of bacterial species or subspecies, but a few studies are available on quantification of 
samples using these techniques. Escoriza et al. used Raman spectroscopy to quantify filtered waterborne bacteria, but ran into problems because of the high fluorescence background from the alumina or silver membrane filters they used [61]. There are also some studies on quantification of viruses using SERS [62, 63].

Raman spectroscopy and, in particular, SERS are sensitive enough to detect bacteria at concentrations considered normal and pathologic for UTIs. This can be verified by performing quantification studies of known bacterial concentrations. For example, E. coli bacteria were cultured and their concentration in solution was determined using their optical density. Samples were diluted serially with sterile deionized water to obtain the following concentrations: $10^{3}, 10^{4}, 10^{5}$, $10^{6}, 10^{7}$, and $10^{8}$ bacteria/mL. $10 \mu \mathrm{L}$ of each sample were mixed with an equal volume of concentrated gold nanoparticles, spotted on glass slides, and allowed to dry. SERS spectra were collected from 22 samples using a portable commercial Raman spectrometer at $785 \mathrm{~nm}$ excitation wavelength and $4.5 \mathrm{~cm}^{-1}$ resolution. Spectra were preprocessed by filtering to remove the background and high-frequency noise. Figure 4 shows the spectra after preprocessing. The feature vector for each sample contained the preprocessed Raman spectrum as well as its first and second derivatives. Each sample was assigned a class of 0 for no infection (concentrations of $\leq 10^{4}$ bacteria/mL) or 1 for infection (concentration $\geq 10^{5}$ bacteria/mL.) The outcome showed that $\sim 82 \%$ of the samples were correctly classified as negative (class 0 ) or positive (class 1) for UTI [18].

The same serial dilutions of $E$. coli bacteria $\left(10^{3}, 10^{4}\right.$, $10^{5}, 10^{6}, 10^{7}$, and $10^{8}$ bacteria/mL) were also studies using a $532 \mathrm{~nm}$ excitation, $3 \mathrm{~cm}^{-1}$ resolution, commercial Raman system. In this case, the bacteria were mixed with silver nanoparticles and spotted on glass slides as before. Spectra were preprocessed by filtering to remove the background and high frequency noise. Figure 5 shows a correlation between the total intensity in the high-wave region of the SERS spectra, from 2900 to $3000 \mathrm{~cm}^{-1}$, with various log concentrations of bacteria indicating an almost linear relationship.

These results are, of course, very preliminary, and much more work is required to develop the technique. They do indicate, however, that it will be possible to examine a urine sample and determine whether it is negative or positive for UTI as well as determine the concentration of bacteria in the urine sample based on its Raman spectral properties. Some preprocessing of urine samples will be done before SERS spectra are collected in order to (a) concentrate samples and make the method more sensitive and (b) remove other types of cells like epithelial or white blood cells that could be present in the sample. Typically, UTIs are caused by a single type of bacteria and it is uncommon to have additional bacterial species in an infected urine culture. A simple 2-step filtration process that would first remove larger, epithelial cells, and WBCs and would secondly concentrate any bacteria on a smaller pore size filter, would allow the isolation of bacteria from urine and the collection of their SERS spectra. In case two or more bacterial species are present in a urine sample the classification algorithm could be trained to detect two or more species at the same time.

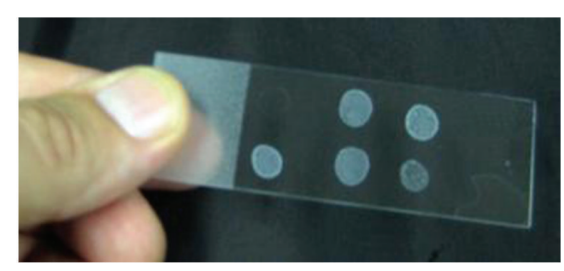

(a)

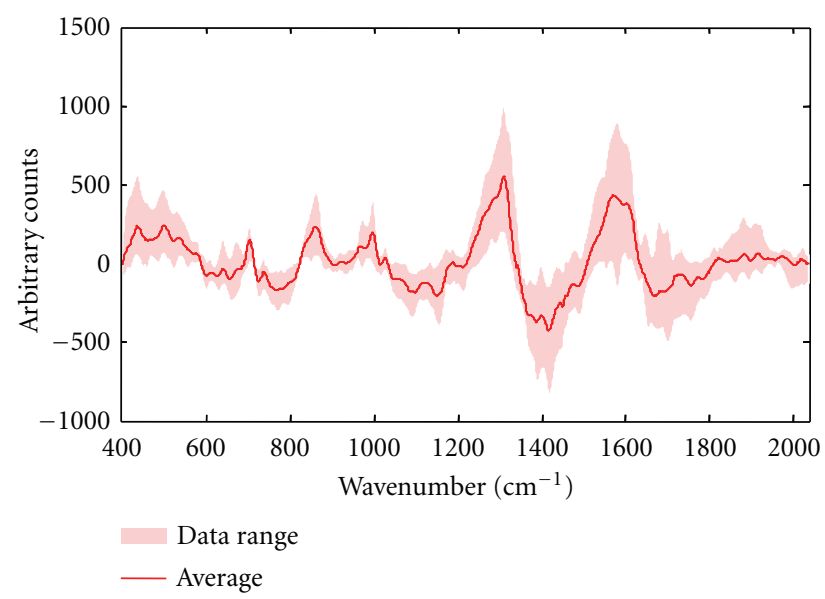

(b)

Figure 4: (a) Bacteria (E. coli) and gold nanoparticles spotted on a glass slide for SERS measurement. (b) SERS spectra for different E. coli samples after preprocessing, indicating the average spectrum and the range of values, that is, intersample variability.

3.2. Identification of the Pathogen in a Positive Sample. As mentioned in the introduction, several investigators are evaluating the use of Raman spectroscopy as a tool for bacterial classification. Recent studies have shown that employing novel feature creation techniques, such as the ratios of different Raman bands (Figure 2) yields good results even when using a low-cost, low-resolution Raman system [17]. The power of Raman spectroscopy for UTI diagnosis can be verified experimentally. For example, clinical bacterial isolates from patients with UTI $(n=75)$, previously identified by conventional tests as positive, were collected from clinical laboratories. Specifically, spectra from 25 samples of E. coli, 25 of K. pneumonia, and 25 of Proteus spp. were acquired using a portable commercial Raman spectrometer at $785 \mathrm{~nm}$ excitation wavelength and $4.5 \mathrm{~cm}^{-1}$ resolution. No filtering of the spectra was performed. Each spectrum was broken up into $25 \mathrm{~cm}^{-1}$ segments and the mean intensity for each segment was determined. Ratios of each segment's mean intensity to every other segment's mean intensity were calculated. The classification method used was Linear Discriminant Analysis with a Principal Components transformation to reduce the dimensionality of the data. A leave-one-out crossvalidation procedure was performed to verify the performance of the classifier. The overall accuracy of the technique was $95 \%$. Very high sensitivity $(88-100 \%)$ and specificity (94-100\%) values for classification of the three species of bacteria were obtained. 


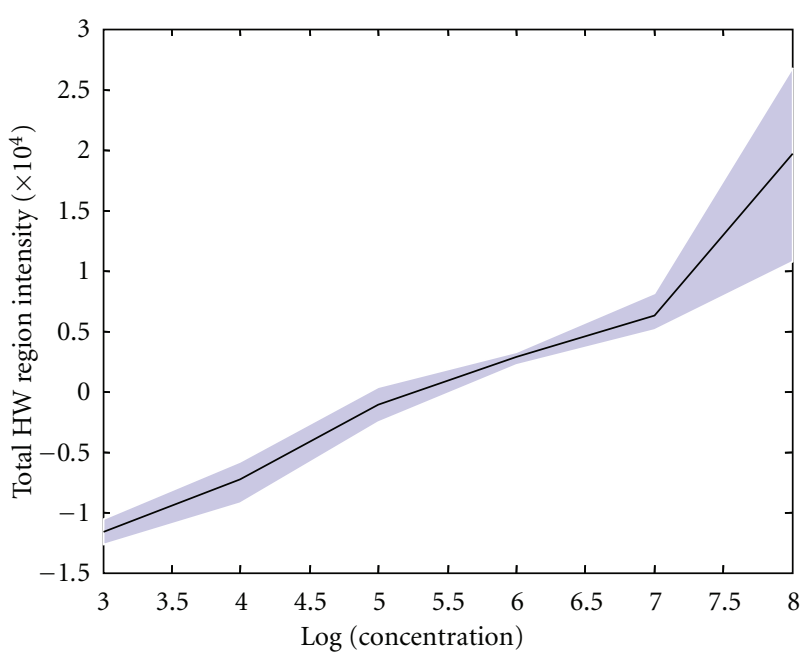

FIgURE 5: Correlation between the total intensity of the 2900 $3000 \mathrm{~cm}^{-1}$ range of the SERS spectra with various concentrations of $E$. coli samples. The shaded region illustrates the range between minimum and maximum numbers, whereas the black line is the mean value.

The use of SERS can further enhance the sensitivity and specificity of bacterial classification providing accurate results for more than three species at a time. To verify this, clinical bacterial isolates from patients with UTI $(n=46)$ were obtained from clinical laboratories after being identified as positive using conventional tests. Specifically, 10 samples of E. coli, 9 of K. pneumoniae, 9 of Proteus spp., 9 of Enterococcus spp., and 9 of Citrobacter spp. were obtained for SERS using the same spectrometer as before. Ratio features, PCA, DA, and a leave-one-out cross-validation procedure were performed as before. Classification was originally done for 3 species (E. coli, K. pneumonia, Proteus spp.) and then for all 5 species of bacteria. Table 1 illustrates the results of the classification analysis of the SERS, spectra and Figure 6 shows two examples of the classification. These results suggest that classification of bacteria belonging to 3 classes using SERS is as accurate (93\%) as classification using normal Raman spectra $(95 \%)$. However, much more accurate classification of bacteria belonging to 5 classes is achieved using SERS (91\%) compared to using normal Raman spectra (73\%). A wavenumber band of $25 \mathrm{~cm}^{-1}$ was proved to be a better choice for providing more accurate classification of the 5 classes of bacteria using the ratios method.

3.3. Antibiotic Sensitivity of Bacterial Samples. In order for the diagnosis of a UTI to be complete, the antibiotic sensitivity of the involved pathogen must be addressed. Most of the studies, in the past, involved the use of Raman for distinguishing live bacteria from bacteria that were killed by heating $[64,65]$, desiccation $[66,67]$, or UV irradiation $[68,69]$. More recent work by Liu et al. [16] shows very clear differences between the spectra of bacteria that are sensitive and the spectra of bacteria that are resistant to an antibiotic. However, no classification of samples as sensitive or resistant to an antibiotic was done by this group. The current
TABLE 1: Results of classification analysis of SERS spectra from 3 or 5 classes of bacteria.

\begin{tabular}{lcc}
\hline Classes & Band size $\left(\mathrm{cm}^{-1}\right)$ & Correct classification \\
\hline 3 & 50 & $28 / 30(93 \%)$ \\
3 & 25 & $28 / 30(93 \%)$ \\
5 & 50 & $40 / 46(87 \%)$ \\
5 & 25 & $42 / 46(91 \%)$ \\
\hline
\end{tabular}

hypothesis is that exposure of bacteria to an antibiotic to which they are sensitive will cause either external or internal molecular changes, depending on the mechanism of action of the antibiotic. It is expected that the short life cycle of bacteria will allow the visualization of these changes in their Raman spectra within a few hours of exposure to an antibiotic.

In a recent study [17], Raman spectra were collected from 27 strains of bacteria, belonging to the species E. coli, K. pneumonia, and Proteus spp., shown to be sensitive to Ciprofloxacin and resistant to Amoxicillin by conventional antibiograms. The spectra were collected after treatment in the presence or absence of each of each of the two antibiotics for 0,2 , and 4 hours. Spectra were collected using a portable, commercial, Raman spectrometer at $785 \mathrm{~nm}$ excitation wavelength and $4.5 \mathrm{~cm}^{-1}$ resolution. The spectra were filtered and the fluorescence background was subtracted. The Raman spectrum as well as the first and second derivatives were included in the feature vector. A principal components (PCs) transformation was used, and only PCs describing the highest variance were retained. The data was, then, analyzed using the MANOVA algorithm which calculated a score for each sample which would provide maximum separation between a group of bacteria which were sensitive to an antibiotic with the groups of bacteria which were resistant or were not treated with antibiotic. The study was repeated using SERS with similar results shown in Figure 7; the MANOVA score of bacteria incubated with Ciprofloxacin is significantly lower than that of untreated bacteria or bacteria treated with Amoxicillin even as early as 2 hours after incubation with the antibiotics. These results suggest that SERS, with increased sensitivity and improved speed compared to Raman, could be used to determine the antibiotic susceptibility of bacteria even after a very short treatment with the antibiotic. These studies are currently being expanded to include more bacteria and more antibiotics.

\section{Conclusion}

In this paper, the current state of the application of Raman spectroscopy in bacterial identification and, more specifically, UTI diagnosis is presented. Raman spectroscopy and SERS, in particular, offer the possibility to develop highly versatile and powerful diagnostic tools. They can provide complex biochemical information which, in conjunction with advanced analysis and classification techniques, can lead to rapid and accurate diagnosis of UTIs. Recent advances in the instrumentation, experimental techniques, analysis, 


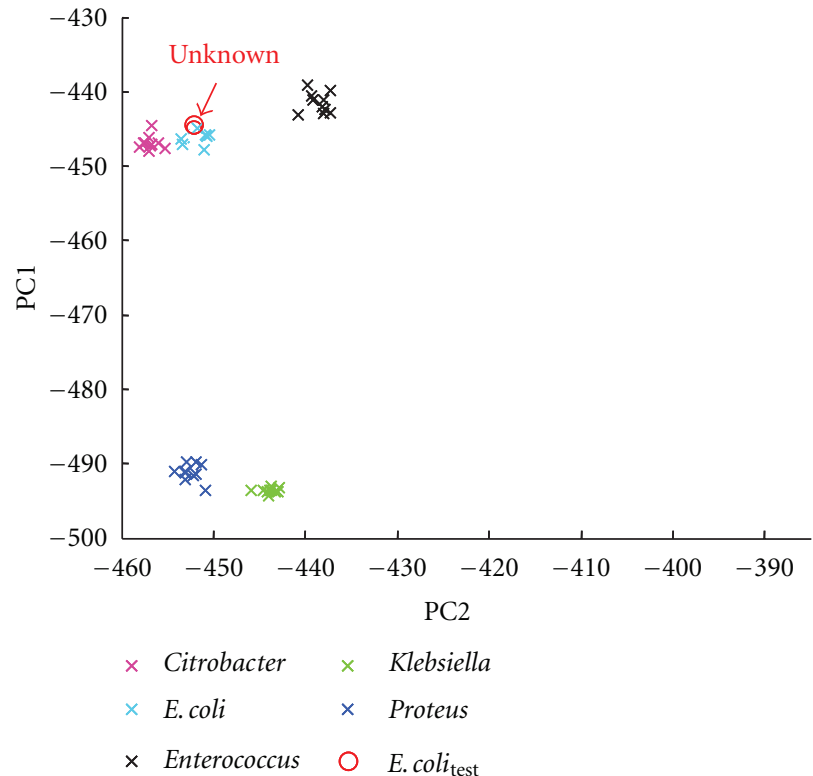

(a)

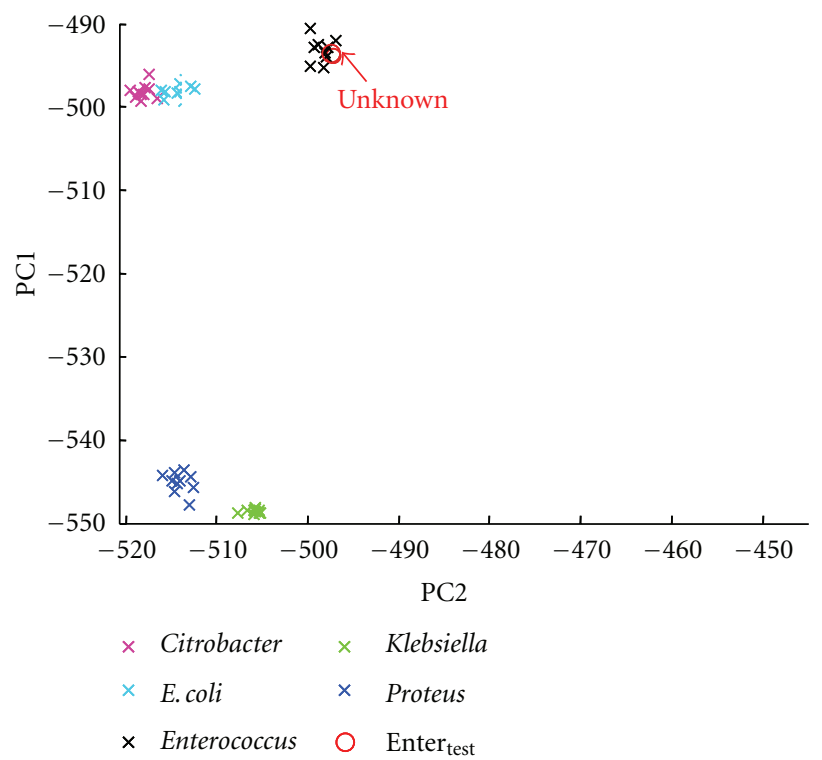

(b)

Figure 6: SERS classification of two unknowns, E. coli, (a) and Enterococcus spp., (b). The first two principal components (PC1 and PC2), that contained the most information, were plotted against each other for visualization purposes. Each of the unknowns clearly falls in the correct species cluster. Note that the plots differ since the training sets differ by one member.

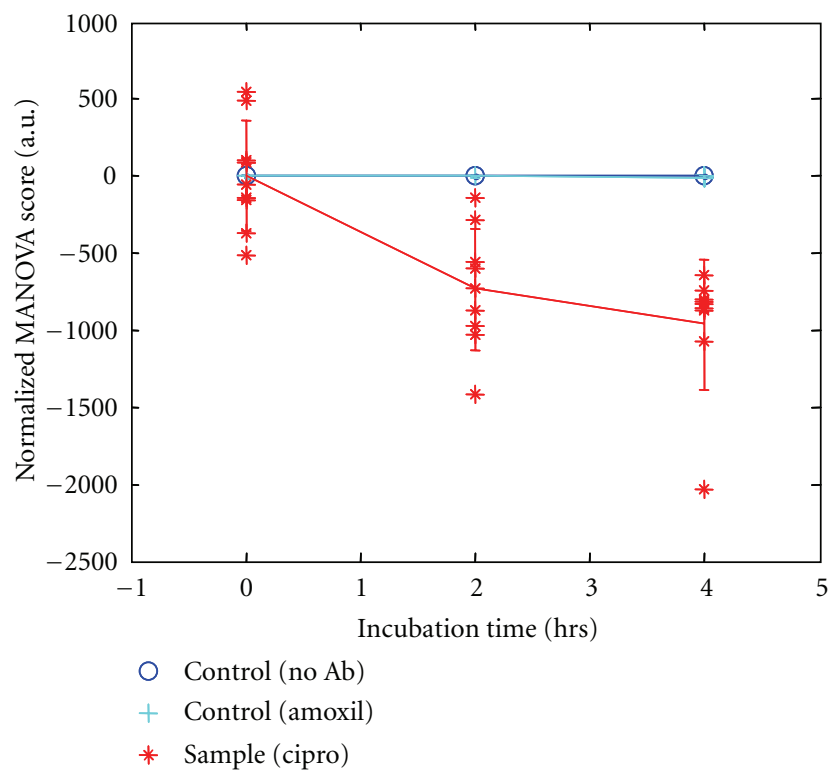

FIgURE 7: Normalized MANOVA score of SERS spectra of E. coli, K. pneumonia, and Proteus spp. strains after their incubation in the presence of $0.128 \mu \mathrm{g} / \mathrm{mL}$ Ciprofloxacin $(*)$, in the presence of $64 \mu \mathrm{g} / \mathrm{mL}$ Amoxicillin $(+)$, or in the absence of any antibiotic $(\bigcirc)$.

and classification have enabled the fast and accurate quantification and identification of bacterial populations as well as the determination of their sensitivity to antibiotics. These results are still preliminary and must be significantly expanded. However, there is great interest in the area and the research community actively strives to improve the yield and accuracy of Raman-based diagnostics. In the future, a simple, point-of-care, device is envisioned which will be able to give a complete UTI diagnosis, from a single urine sample, within a few hours. Such a technology could have significant shortand long-term benefits for public health by reducing the cost of diagnosis, improving prognosis, and reducing the un- 
necessary use of antibiotics and, therefore, bacterial resistance.

\section{Acknowledgments}

Some of the research presented in this paper was partially supported by the KIOS Research Center for Intelligent Systems and Networks of the University of Cyprus and the Research Promotion Foundation of Cyprus. The authors also wish to thank all their collaborators at P. Hadjikoumis Microbiology Laboratory and the Limassol General Hospital for their support.

\section{References}

[1] T. L. Griebling, "Urinary tract infection in women," in Urologic Diseases in America, pp. 587-619, DHHS, PHS, NIH, NIDDK, NIH, Washington, DC, USA, 2007.

[2] T. L. Griebling, "Urinary tract infection in women," in Urologic Diseases in America, pp. 621-645, DHHS, PHS, NIH, NIDDK, NIH, Washington, DC, USA, 2007.

[3] National Center for Health Statistics, National Hospital Discharge Survey: 2004 Annual Summary With Detailed Diagnosis and Procedure Data, DHHS, Centers for Disease Control and Prevention, DHHS, Hyattsville, Md, USA, 2006, 1733.

[4] M. S. Litwin and C. S. Saigal, "Introduction," in Urologic Diseases in America, pp. 3-7, DHHS, PHS, NIH, NIDDK, NIH, Washington, DC, USA, 2007.

[5] R. Gruneberg, "Changes in urinary pathogens and their antibiotic sensitivities, 1971-1992," Journal of Antimicrobial Chemotherapy, vol. 33, supplement A, pp. 1-8, 1994.

[6] A. Casadevall, "Crisis in infectious diseases: time for a new paradigm?” Clinical Infectious Diseases, vol. 23, no. 4, pp. 790794, 1996.

[7] S. Cosgrove and Y. Carmeli, "The impact of antimicrobial resistance on health and economic outcomes," Clinical Infectious Diseases, vol. 36, no. 11, pp. 1433-1437, 2003.

[8] A. Alanis, "Resistance to antibiotics: are we in the postantibiotic era?" Archives of Medical Research, vol. 36, no. 6, pp. 697-705, 2005.

[9] E. Mothershed and A. Whitney, "Nucleic acid-based methods for the detection of bacterial pathogens: present and future considerations for the clinical laboratory," Clinica Chimica Acta, vol. 363, no. 1-2, pp. 206-220, 2006.

[10] J. Rolain, M. Mallet, P. Fournier, and D. Raoult, "Real-time PCR for universal antibiotic susceptibility testing," Journal of Antimicrobial Chemotherapy, vol. 54, no. 2, pp. 538-541, 2004.

[11] W. Chen, P. Tsai, and Y. Chen, "Functional nanoparticlebased proteomic strategies for characterization of pathogenic bacteria," Analytical Chemistry, vol. 80, no. 24, pp. 9612-9621, 2008.

[12] K. Maquelin, L. Choo-Smith, T. Vreeswijk et al., "Raman spectroscopic method for identification of clinically relevant microorganisms growing on solid culture medium," Analytical Chemistry, vol. 72, no. 1, pp. 12-19, 2000.

[13] K. C. Schuster, I. Reese, E. Urlaub, J. R. Gapes, and B. Lendl, "Multidimensional information on the chemical composition of single bacterial cells by confocal Raman microspectroscopy," Analytical Chemistry, vol. 72, no. 22, pp. 5529-5534, 2000.

[14] K. C. Schuster, E. Urlaub, and J. R. Gapes, "Single-cell analysis of bacteria by Raman microscopy: spectral information on the chemical composition of cells and on the heterogeneity in a culture," Journal of Microbiological Methods, vol. 42, no. 1, pp. 29-38, 2000.

[15] K. Kneipp, M. Moskovits, and H. Kneip, "Surface-enhanced Raman scattering," Physics and Applications Topics in Applied Physics, vol. 103, pp. 1-464, 2006.

[16] T. T. Liu, Y. H. Lin, C. S. Hung et al., "A high speed detection platform based on surface-enhanced Raman scattering for monitoring antibiotic-induced chemical changes in bacteria cell wall," Plos One, vol. 4, no. 5, Article ID e5470, 2009.

[17] E. Kastanos, A. Kyriakides, K. Hadjigeorgiou, and C. Pitris, "A novel method for urinary tract infection diagnosis and antibiogram using Raman spectroscopy," Journal of Raman Spectroscopy, vol. 41, no. 9, pp. 958-963, 2010.

[18] E. Kastanos, K. Hadjigeorgiou, A. Kyriakides, and C. Pitris, "Classification of bacterial samples as negative or positive for a UTI and antibiogram using surface enhanced Raman spectroscopy," in Proceedings of the SPIE, vol. 7911, 2011.

[19] J. Magee, "Whole-organism fingerprinting," in Handbook of New Bacterial Systematics, M. Goodfellow and A. O'Donnell, Eds., pp. 383-427, Academic Press, London, UK, 1993.

[20] A. Mahadevan-Jansen, "Raman spectroscopy from benchtop to bedside," in Biomedical Photonics Handbook, T. Vo-Dinh, Ed., CRC Press, London, USA, 2002.

[21] O. Preisner, J. Lopes, and J. Menezes, "Uncertainty assessment in FT-IR spectroscopy based bacteria classification models," Chemometrics and Intelligent Laboratory Systems, vol. 94, no. 1, pp. 33-42, 2008.

[22] J. Forrester, N. Valentine, Y. Su, and T. Johnson, "Chemometric analysis of multiple species of Bacillus bacterial endospores using infrared spectroscopy: discrimination to the strain level," Analytica Chimica Acta, vol. 651, no. 1, pp. 24-30, 2009.

[23] R. Jarvis and R. Goodacre, "Ultra-violet resonance Raman spectroscopy for the rapid discrimination of urinary tract infection bacteria," FEMS Microbiology Letters, vol. 232, no. 2, pp. 127-132, 2004.

[24] D. Hutsebaut, K. Maquelin, P. De Vos, P. Vandenabeele, L. Moens, and G. Puppels, "Effect of culture conditions on the achievable taxonomic resolution of Raman spectroscopy disclosed by three Bacillus species," Analytical Chemistry, vol. 76, no. 21, pp. 6274-6281, 2004.

[25] C. Xie, J. Mace, M. Dinno et al., "Identification of single bacterial cells in aqueous solution using confocal laser tweezers Raman spectroscopy," Analytical Chemistry, vol. 77, no. 14, pp. 4390-4397, 2005.

[26] M. Harz, P. Rösch, K. Peschke, O. Ronneberger, H. Burkhardt, and J. Popp, "Micro-Raman spectroscopic identification of bacterial cells of the genus Staphylococcus and dependence on their cultivation conditions," Analyst, vol. 130, no. 11, pp. 1543-1550, 2005.

[27] U. Schmid, P. Rosch, M. Krause, M. Harz, J. Popp, and K. Baumann, "Gaussian mixture discriminant analysis for the single-cell differentiation of bacteria using micro-Raman spectroscopy," Chemometrics and Intelligent Laboratory Systems, vol. 96, no. 2, pp. 159-171, 2009.

[28] K. Kneipp, Y. Wang, H. Kneipp et al., "Single molecule detection using surface-enhanced Raman scattering (SERS)," Physical Review Letters, vol. 78, no. 9, pp. 1667-1670, 1997.

[29] S. Nie and S. Emory, "Probing single molecules and single nanoparticles by surface-enhanced Raman scattering," Science, vol. 275, no. 5303, pp. 1102-1106, 1997.

[30] M. Moskovits, "Surface-enhanced spectroscopy," Reviews of 
Modern Physics, vol. 57, no. 3, pp. 783-826, 1985.

[31] T. Vo-Dinh, F. Yan, and D. Stokes, "Plasmonics-based nanostructures for surface-enhanced Raman scattering bioanalysis," Methods in Molecular Biology, vol. 300, pp. 255-283, 2005.

[32] R. Jarvis, N. Law, I. Shadi, P. O’Brien, J. Lloyd, and R. Goodacre, "Surface-enhanced Raman scattering from intracellular and extracellular bacterial locations," Analytical Chemistry, vol. 80, no. 17, pp. 6741-6746, 2008.

[33] S. Efrima and B. Bronk, "Silver colloids impregnating or coating bacteria," Journal of Physical Chemistry B, vol. 102, no. 31, pp. 5947-5950, 1998.

[34] L. Zeiri, B. V. Bronk, Y. Shabtai, J. Czege, and S. Efrima, "Silver metal induced surface enhanced Raman of bacteria," Colloids and Surfaces A, vol. 208, no. 1-3, pp. 357-362, 2002.

[35] L. Zeiri, B. Bronk, Y. Shabtai, J. Eichler, and S. Efrima, "Surface-enhanced raman spectroscopy as a tool for probing specific biochemical components in bacteria," Applied Spectroscopy, vol. 58, no. 1, pp. 33-40, 2004.

[36] S. Efrima and L. Zeiri, "Understanding SERS of bacteria," Journal of Raman Spectroscopy, vol. 40, no. 3, pp. 277-288, 2009.

[37] R. Jarvis and R. Goodacre, "Discrimination of bacteria using surface-enhanced Raman spectroscopy," Analytical Chemistry, vol. 76, no. 1, pp. 40-47, 2004.

[38] R. Jarvis, A. Brooker, and R. Goodacre, "Surface-enhanced Raman spectroscopy for bacterial discrimination utilizing a scanning electron microscope with a Raman spectroscopy interface," Analytical Chemistry, vol. 76, no. 17, pp. 5198-5202, 2004.

[39] R. Jarvis, A. Brooker, and R. Goodacre, "Surface-enhanced Raman scattering for the rapid discrimination of bacteria," Faraday Discussions, vol. 132, pp. 281-292, 2006.

[40] R. Jarvis and R. Goodacre, "Characterisation and identification of bacteria using SERS," Chemical Society Reviews, vol. 37, no. 5, pp. 931-936, 2008.

[41] W. Premasiri, D. Moir, M. Klempner, N. Krieger, G. Jones, and L. Ziegler, "Characterization of the surface enhanced Raman scattering (SERS) of bacteria," Journal of Physical Chemistry B, vol. 109, no. 1, pp. 312-320, 2005.

[42] I. Patel, W. Premasiri, D. Moir, and L. Ziegler, "Barcoding bacterial cells: a SERS-based methodology for pathogen identification," Journal of Raman Spectroscopy, vol. 39, no. 11, pp. 1660-1672, 2008.

[43] S. Hennigan, J. Driskell, R. Dluhy et al., "Detection of Mycoplasma pneumoniaein simulated and true clinical throat swab specimens by nanorod array-surface-enhanced raman spectroscopy," Plos One, vol. 5, no. 10, Article ID e13633, 2010.

[44] A. Walter, A. Marz, W. Schumacher, P. Rosch, and J. Popp, "Towards a fast, high specific and reliable discrimination of bacteria on strain level by means of SERS in a microfluidic device," Lab on a Chip, vol. 11, no. 6, pp. 1013-1021, 2011.

[45] A. Kyriakides, E. Kastanos, K. Hadjigeorgiou, and C. Pitris, "Classification of Raman spectra using the correlation kernel," Journal of Raman Spectroscopy, vol. 42, no. 5, pp. 904-909, 2011.

[46] F. Ehrentreich and L. Summchen, "Spike removal and denoising of Raman spectra by wavelet transform methods," Analytical Chemistry, vol. 73, no. 17, pp. 4364-4373, 2001.

[47] T. Dörfer, W. Schumacher, N. Tarcea, M. Schmitt, and J. Popp, "Quantitative mineral analysis using Raman spectroscopy and chemometric techniques," Journal of Raman Spectroscopy, vol. 41, no. 6, pp. 684-689, 2010.
[48] I. T. Jollife, Principal Component Analysis, Springer, New York, NY, USA, 2nd edition, 2002.

[49] I. T. Jolliffe, "A note on the use of principal components in regression," Journal of the Royal Statistical Society C, vol. 31, no. 3, pp. 300-303, 1982.

[50] J. R. Ferraro, K. Nakamoto, and C. W. Brown, Introductory Raman Spectroscopy, Elsevier Science, New York, NY, USA, 2nd edition, 2003.

[51] Y. L. Loethen, D. Zhang, R. N. Favors, S. B. G. Basiaga, and D. Ben-Amotz, "Second-derivative variance minimization method for automated spectral subtraction," Applied Spectroscopy, vol. 58, no. 3, pp. 272-278, 2004.

[52] N. Navas, J. Romero-Pastor, E. Manzano, and C. Cardell, "Raman spectroscopic discrimination of pigments and tempera paint model samples by principal component analysis on firstderivative spectra," Journal of Raman Spectroscopy, vol. 41, no. 11, pp. 1196-1203, 2010.

[53] F. B. Baker and L. J. Hubert, "Measuring the power of hierarchical cluster analysis," Journal of the American Statistical Association, vol. 70, no. 349, pp. 31-38, 1975.

[54] W. J. Krzanowski, Principles of Multivariate Analysis: a User's Perspective, Oxford University Press, New York, NY, USA, 2000.

[55] M. Barker and W. Rayens, "Partial least squares for discrimination," Journal of Chemometrics, vol. 17, no. 3, pp. 166-173, 2003.

[56] J. Weston and Watkins, "Support vector machines for multiclass pattern recognition," in Proceedings of the Seventh European Symposium on Artificial Neural Networks, Brussels, Belgium, April 1999.

[57] J. A. Westerhuis, H. C. J. Hoefsloot, S. Smit et al., "Assessment of PLSDA cross validation," Metabolomics, vol. 4, no. 1, pp. 81$89,2008$.

[58] V. N. Vapnik, Statistical Learning Theory, Wiley-Interscience, New York, NY, USA, 1998.

[59] B. Schölkopf, C. J. C Burges, and A. J. Smola, Eds., Advances in Kernel Methods-Support Vector Learning, MIT Press, Cambridge, Mass, USA, 1998.

[60] N. Cristianini and J. Shawe-Taylor, An Introduction to Support Vector Machines: and other Kernel-Based Learning Methods, Cambridge University Press, Cambridge, UK, 2004.

[61] M. Escoriza, J. VanBriesen, S. Stewart, J. Maier, and P. Treado, "Raman spectroscopy and chemical imaging for quantification of filtered waterborne bacteria," Journal of Microbiological Methods, vol. 66, no. 1, pp. 63-72, 2006.

[62] C. Fan, Z. Hu, L. Riley, G. Purdy, A. Mustapha, and M. Lin, "Detecting food-and waterborne viruses by surfaceenhanced raman spectroscopy," Journal of Food Science, vol. 75, no. 5, pp. M302-M307, 2010.

[63] X. Wang, Y. Li, H. Wang et al., "Gold nanorod-based localized surface plasmon resonance biosensor for sensitive detection of hepatitis B virus in buffer, blood serum and plasma," Biosensors and Bioelectronics, vol. 26, no. 2, pp. 404-410, 2010.

[64] M. Baek, W. Nelson, D. Britt, and J. Sperry, "UV-excited resonance raman spectra of heat denatured lysozyme and staphylococcus epidermidis," Applied Spectroscopy, vol. 42, no. 7, pp. 1312-1314, 1988.

[65] A. Grow, L. Wood, J. Claycomb, and P. Thompson, "New biochip technology for label-free detection of pathogens and their toxins," Journal of Microbiological Methods, vol. 53, no. 2, pp. 221-233, 2003. 
[66] L. Robertson, A. Campbell, and H. Smith, "Survival of Cryptosporidium parvum oocysts under various environmental pressures," Applied and Environmental Microbiology, vol. 58, no. 11, pp. 3494-3500, 1992.

[67] M. Deng and D. Cliver, "Cryptosporidium parvum studies with dairy products," International Journal of Food Microbiology, vol. 46, no. 2, pp. 113-121, 1999.

[68] M. Lorenzo-Lorenzo, M. Ares-Mazas, I. Villacorta-Martinez de Maturana, and D. Duran-Oreiro, "Effect of ultraviolet disinfection of drinking water on the viability of Cryptosporidium parvum oocysts," Journal of Parasitology, vol. 79, no. 1, pp. 67-70, 1993.

[69] M. Belosevic, S. A. Craik, J. L. Stafford, N. F. Neumann, J. Kruithof, and D. W. Smith, "Studies on the resistance/reactivation of Giardia muris cysts and Cryptosporidium parvum oocysts exposed to medium-pressure ultraviolet radiation," FEMS Microbiology Letters, vol. 204, no. 1, pp. 197-203, 2001. 


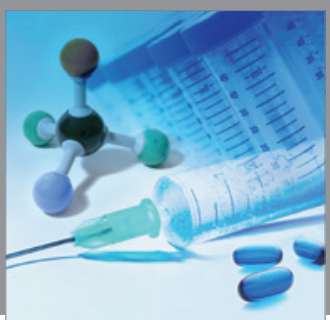

International Journal of

Medicinal Chemistry

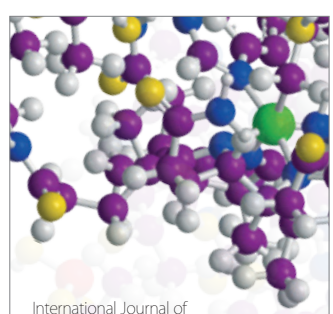

Carbohydrate Chemistry

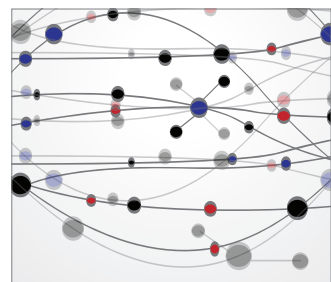

The Scientific World Journal
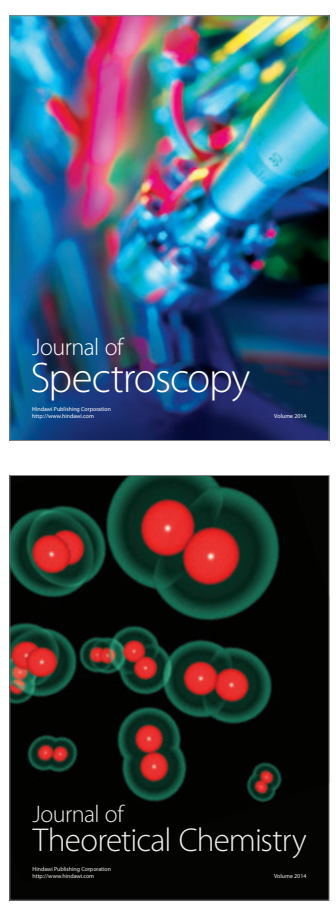
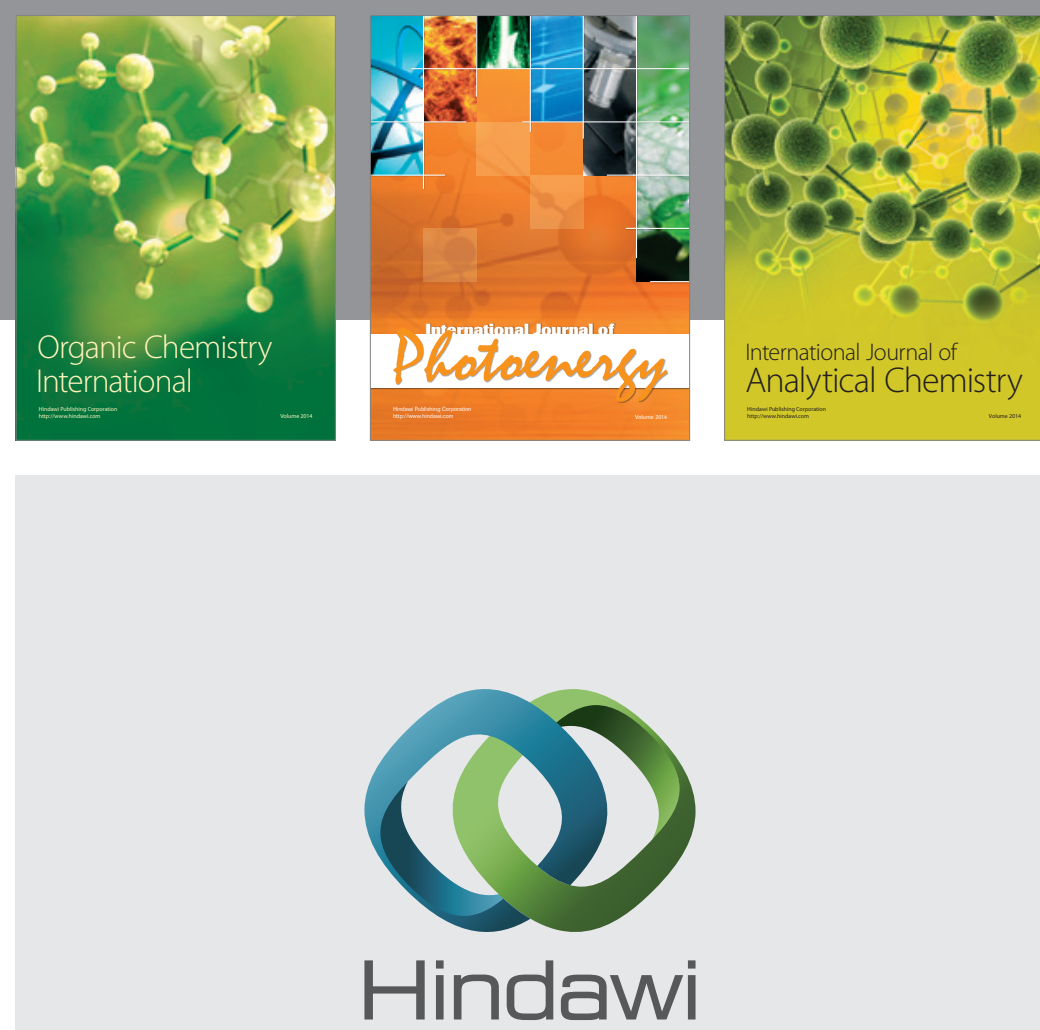

Submit your manuscripts at

http://www.hindawi.com
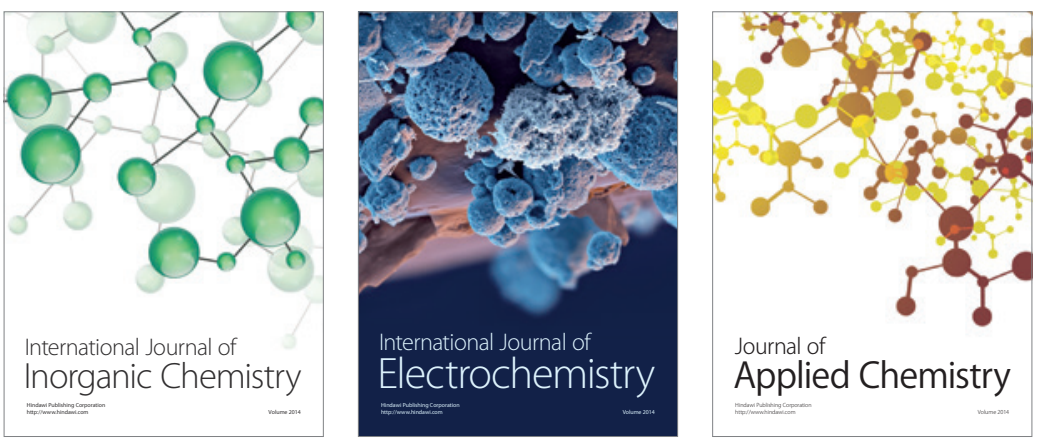

Journal of

Applied Chemistry
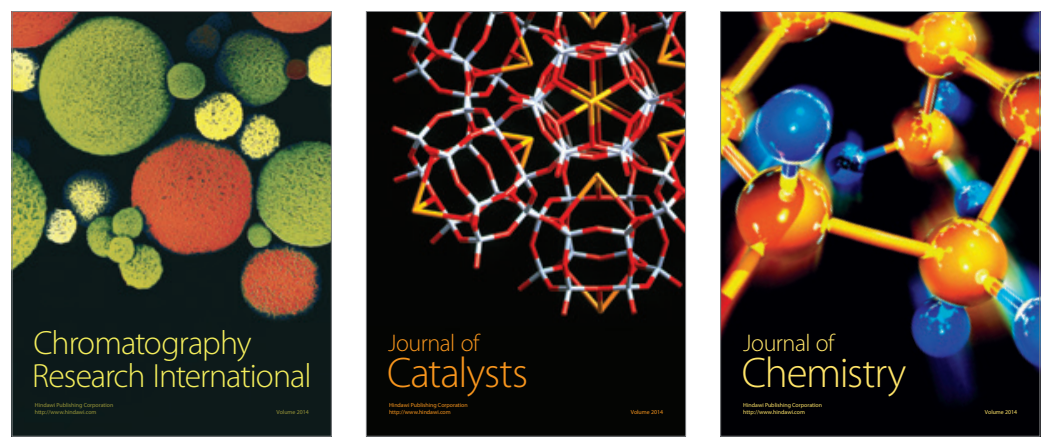
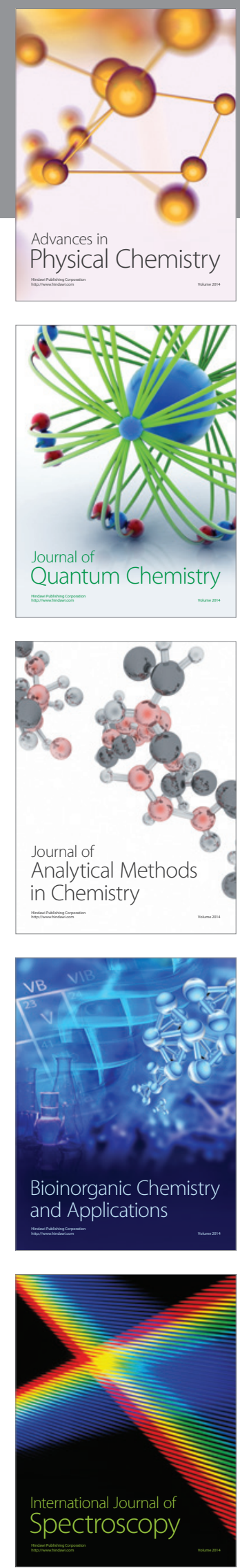